\title{
Astrophysical Fine Tuning, Naturalism, and the Contemporary Design Argument
}

\author{
Mark A. Walker and Milan M. Ćirković
}

Evidence for instances of astrophysical 'fine tuning' (or 'coincidences') is thought by some to lend support to the design argument (i.e. the argument that our universe has been designed by some deity). We assess some of the relevant empirical and conceptual issues. We argue that astrophysical fine tuning calls for some explanation, but this explanation need not appeal to the design argument. A clear and strict separation of the issue of anthropic fine tuning on one hand and any form of Eddingtonian numerology and teleology on the other, may help clarify arguably the most significant issue in the philosophy of cosmology.

\section{Introduction: Fine Tuning in Astrophysics}

Suppose you were asked to be the architect of a new universe- a heady responsibility to be sure. The only stipulation is that your universe must have the same fundamental constants as our universe - the coupling constants for gravity, electromagnetism, weak and nuclear force, as well as quantities such as $c, \mathrm{G}$, or $\eta$, and (possibly) a few cosmological parameters such as the total energy density $\Omega$ or the initial entropy-per-baryon $S$ (of course, the dimensional quantities among these ought to be expressed in terms of dimensionless combinations, like the fine-structure constant $\alpha$ ). However, you are allowed latitude in what values you assign to these fundamental constants. One way to proceed would be to assign these values by consulting a random number table. Using this procedure may speed your task along, but the resulting universe is not likely to be very 'appealing'. The result might be somewhat analogous to using a random number

\footnotetext{
Mark Walker is a Research Associate at Trinity College, University of Toronto, and an assistant professor at the Department of Philosophy, McMaster University, Canada.

Milan M. Ćirković is a Researcher at the Astronomical Observatory Belgrade, Serbia and Montenegro.

Correspondence to: Mark A. Walker, Trinity College, University of Toronto, 15 Devonshire Place, Toronto, Ontario M5S 1H8, Canada. E-mail: walkmar@mcmaster.ca
} 
table to determine the relative amounts of the ingredients when baking a cake. If you choose to be a little more energetic, there are at least a couple of ways in which you could 'improve' your universe: you could provide it with some mathematical orderliness, or perhaps your concern might be to make it hospitable for sentient life. The former idea we will refer to as the idea of 'mathematical fine tuning' and the latter 'anthropic fine tuning'.

Astrophysicists (and others) have applied both of these ideas to our universe; but not always in a manner that makes it clear what is at issue. That there is some distinction to be drawn between these two types of fine tuning may seem obvious, but a recent article critical of the idea of astrophysical fine tuning in a well-known journal (Klee 2002) demonstrates the need to underscore this distinction. Klee believes that he has shown that a 'strong scepticism' about astrophysical fine tuning is warranted. However, he does not distinguish between mathematical and anthropic fine tuning. Consider, in this connection, Klee's understanding of Carr and Rees's (1979) classic paper. According to Klee, the 'chief motivating factor' for Carr and Rees is 'curiosity and the search for mathematical order'. However, this is to profoundly misunderstand Carr and Rees's groundbreaking work. Their study is one that looks to the relation between cosmological parameters and the existence of observers, that is, to anthropic rather than mathematical fine tuning. Klee's article is a prototype of an entire cottage industry of studies critical of the anthropic reasoning, but based upon confusing and/or conflating two different concepts of fine tuning (among other examples, one may mention Barrow and Tipler 1986; Maynard-Smith and Szathmáry 1996; Manson 2000; Mosterín 2000, 2005).

Our aim here is to attempt to disentangle these (and related) ideas as well as assess their applicability to our universe. In the next section, we explore further the ideas of different types of fine tuning. In Section 3, we examine the evidence for fine tuning in our universe. We argue that while there is little empirical evidence for mathematical fine tuning of the universe, there is good (preliminary) evidence that our universe is anthropically fine-tuned. In Section 4, we consider the question of how to explain fine tuning. We argue that there are several explanations that do not invoke the machinations of a deity, including a promising naturalistic explanation for the anthropic fine tuning of our universe that invokes the idea of a multiverse. One upshot of this, as we hope to show, is that it is possible to explain anthropic fine tuning without appealing to some version of the Design argument that explains anthropic fine tuning in terms of the intentions of an architect of our universe. ${ }^{1}$

\section{Types of Fine Tuning}

One of our aims is to show that the notion of 'astrophysical fine tuning' is often understood in a way that invites further clarification. One ambiguity turns on the observational and explanatory tasks associated with the investigation of fine tuning. To illustrate this difference, imagine that two astronomers work side by side for years charting distant galaxies. A certain amount of consensus emerges after their hard labour: they agree that the universe appears to be designed but disagree on the explanation why. One 
thinks it is a matter of some naturalistic process that the universe appears to be designed. The second believes that the appropriate explanation lies in fact that there is a Designer of our universe. What we should say here is that the astronomers agree that the universe is fine-tuned, but disagree on the explanation. Thus, we shall understand the idea of 'fine tuning' as making no commitment to any particular explanation or theory about the nature of the astrophysical fine tuning. So, to the extent that we can distinguish between the activities of observing and theorizing, a commitment to fine tuning is to make an observational claim, e.g. the universe (or some part of the universe) appears to be designed, whereas to invoke a particular explanation for astrophysical fine tuning is to move more into the realm of theory. This distinction is not without parallel: biologists at the end of the 19th century often agreed that organisms appear 'fine-tuned', e.g. that various biological systems of organisms appear to exhibit a great degree of design, but disagreed on the explanation for this observation. Some championed the new Darwinian naturalistic explanation for these observations; others defended the traditional view that these observations were best explained in terms of the work of a Designer. Now, it must be admitted that our use of 'fine tuning' here does not always agree with its use in the literature, since some authors tend to lump what we are terming the 'observational' and 'theoretical' tasks together; however, at least for our purposes here, it will be best to keep these activities distinct.

As intimated above, a further ambiguity can be found among various different types of astrophysical fine tuning, and thus, we want to urge the view that 'astrophysical fine tuning' ought to be seen as a 'genus' which includes various species, among them mathematical and anthropic fine tuning. Let us consider first the case of mathematical fine tuning. The idea that the universe is characterized by mathematical orderliness as a design constraint is an ancient one: it is a doctrine associated with Pythagoras and his followers, and it found its most famous expression in the Ancient world in Plato's Timaeus. Hermann Weyl (1919) might be thought of as the modern progenitor of the idea of mathematical fine tuning. Weyl noticed a convergence on the number $10^{40}$ from two quite different sources. According to Weyl, one arrives at $10^{40}$ when relating electromagnetism and gravity. Specifically, by taking the ratio of strength of an electron's electromagnetic force and the strength of gravitational force as a function of its mass, one arrives at the pure number of $10^{40}$ (the units of measure cancel). The same large number $10^{40}$ is also derived when the radius of the observable universe is expressed in fundamental 'atomic units' of Bohr's radius. Building on the work of Weyl, Eddington (1923) began to see cosmic coincidences converging on (or near) three classes: the number $1,10^{40}$, and those that are a power of $10^{40}$ (like its square, $10^{80}$, etc.). Certainly, if Eddington is right about the convergence of these three classes of numbers, then our universe probably exhibits more mathematical orderliness than the 'random universes' where the constants are assigned according to a random number table. The notion of mathematical fine tuning appeals ultimately to a 'mathematical aesthetic' criterion for universe creation: the idea of mathematical orderliness. An obvious question here is: exactly what is 'mathematical orderliness'? We shall work with the intuitive idea that some universes might exhibit more mathematical elegance than others, as well as discussing below what we take to be clear examples of this idea, while putting aside the 
seemingly difficult problem of a detailed analysis of exactly what this notion amounts to. (We conjecture that the problem is difficult, since it looks to be on par with other well-known problems in the philosophy of science such as explicating the notion of 'simplicity'. Thus, it is often suggested that, other things being equal, we ought to prefer the theory that is simpler. However, philosophers of science have had a difficult time spelling out exactly what the concept simplicity amounts to. So the thought is that articulating the idea of mathematical fine tuning may present similar difficulties.)

Weyl and Eddington tended to look at the very large and small features of our universe in their attempts to show that the universe is mathematically fine-tuned. However, it is worth noting that 'medium-sized' astrophysical objects, too, might exhibit mathematical fine tuning. Imagine the Prime Number Universe (PNU). The PNU is a counterfactual universe where planetary systems are based on prime numbers such that the number of planets in a solar system is always a prime number. In addition, every planet has a prime number of moons arranged such that planets with larger orbits have a larger prime number of moons. Everything else, including values of fundamental constants of nature, stay the same in PNU as in our observable universe. To get a fix on this, imagine what one would have to do to make our solar system into a member of the PNU: we might have to destroy the last two planets so that we have a prime number of seven in our solar system. Furthermore, we might add and subtract a few moons to yield the following pattern: Mercury would have two moons, Venus three, Earth five, Mars seven, Jupiter 11, Saturn 13, and Uranus 17. If modern astronomy had discovered that our solar system had this exact number of planets and moons, then we would have been in an excellent position to say that the solar system is mathematically fine-tuned. ${ }^{2}$ If we think about a future when we are able to observe other planetary systems in more detail, we might imagine evidence mounting that the universe as a whole is as described by the PNU: every other planetary system we examine has this same prime number configuration corroborating the PNU conjecture. PNU is quite fanciful, but it does illustrate that mathematically, fine tuning could, in principle, be based on observations quite different from the relations of the fundamental constants.

Turning now to the idea of anthropic fine tuning, we would do well to remind ourselves that this notion is related to the concept of an 'anthropic principle', ${ }^{3}$ a term which comes from Carter (1974) and refers to the idea that the values of fundamental constants of our universe are (purportedly) highly constrained by the contingent fact that human observers exist: what might otherwise seem to be possible values for the fundamental constants can be ruled out because they are inconsistent with (say) the existence of stars that are stable for billions of years, or with the existence of galaxies or supernovas. The 'anthropic thinking' here is that both stable stars and supernovas are necessary for the evolution of intelligent life as we know it: the former as a home and energy source for life to evolve, the latter to produce the heavier elements in the periodic table necessary for many life processes. ${ }^{4}$ If Carter and others are right, the chance that a 'random universe' would be hospitable for humans is astronomically smallalmost all 'random universes' would be inhospitable to human life. The commitment to anthropic fine tuning then is that our universe appears to be designed to be hospitable for human life. As is the case with mathematical fine tuning, the commitment to 
anthropic fine tuning is taken to be distinct from the explanatory question of how we should explain this observation. That is, should it be explained in terms of a Designer, or is there some naturalistic explanation for this?

Before looking at the evidence for astrophysical fine tuning, we should ask: are these two types of fine tuning jointly exhaustive? As far as serious astrophysical research goes, the answer seems to be yes. The literature may sometimes run these two types of fine tuning together but, to the best of our knowledge, all serious research can be lumped under one of these two types of fine tuning. Clearly, there are other logically possible types of fine tuning. In the past, it was thought that the universe was organized along aesthetic lines: the stars in the sky were said to represent various objects, e.g. 'Orion's Belt' and the 'Big Dipper' are configurations of stars that are said to represent familiar human objects. To the extent that one took seriously the idea that the stars are pictures of such things, then one might say that the universe is 'aesthetically fine-tuned'. Imagine if the stars in the night sky formed a perfect outline of the young Elvis: we would have good reason to say that the universe was aesthetically fine-tuned (by a deistic Elvis fan). In fact, there are a number of ways that a universe in theory could be fine-tuned. Imagine we discover that all the visible galaxies form an intricate pattern that looks exactly like a person slipping on a banana peel. Here, we might think that the universe is humorously fine-tuned (to the extent that one finds 'Jerry Lewis'-style humour humorous). The possibilities here are perhaps endless: in theory, we could discover that the universe appeared to be designed to maximize the production of supernovas, earthsized planets, a certain element on the period table, animal flesh, etc. While it would be wrong to dismiss any of these exotic possibilities a priori, the previous point stands: the only two serious contenders in the field (at the moment) are mathematical fine tuning and anthropic fine tuning; so we shall confine our attention to them.

Are these two types of fine tuning mutually exclusive or mutually entailing? At least in the weak sense of what is logically possible, a negative verdict seems indicated, that is, it seems that the two concepts are logically independent. It seems that there is no a priori reason to suppose that there is, or there is not, overlap between the set of universes that exhibit mathematical fine tuning and the set of universes that exhibit anthropic fine tuning. In other words, there is nothing about the concept of mathematical fine tuning that entails that all or any of the set of mathematically fine-tuned universes might be hospitable to human life; nor does the concept of anthropic fine tuning entail the concept of mathematical fine tuning. It seems perfectly conceivable that there might be universes that exhibit a high degree of mathematical fine tuning without being hospitable to human life. For instance, we could have a world in which the fundamental physical and mathematical constants exactly satisfy a numerologically appealing relationship:

The Beautiful Theorem: $\alpha=\pi^{1} e^{4} c^{9} h^{16} G^{25} \Omega^{36}$.

But there is no reason to suppose that a universe that conforms to the Beautiful Theorem would support human life! So, we can easily imagine a universe that exhibits all sorts of Eddington numerology, but which is inhospitable to life (imagine radiation 
levels too high to allow life to develop). On the other hand, we can imagine a universe that is delicately balanced in its fundamental constituents for human life, but does not exhibit the mathematical orderliness of a mathematically fine-tuned universe. On the other hand, there is no reason to suppose that the sets must be mutually exclusive: it may well be that many universes that are hospitable to human life exhibit mathematical orderliness. So, a commitment to one form of fine tuning does not, a priori, imply or exclude a commitment to the other.

But while the two concepts of fine tuning are logically independent, it may well be that the two concepts are nomologically related. The idea here is that, while as a matter of logic the two notions are independent, they are related in terms of some physical laws, just as it is logically possible that the reader of this paper is not attracted to its authors, but as a nomological necessity there is an attraction: a gravitational attraction. Similarly, we cannot rule out a priori the possibility that there are nomological relations between anthropic fine tuning and mathematical fine tuning. One possibility is advances in physics, for example, the currently sought 'Theory of Everything', might reveal that mathematical fine tuning and anthropic fine tuning are related in some lawlike fashion. (We will discuss this possibility below.)

\section{Evidence for Astrophysical Fine Tuning}

In this section, we argue that there is little empirical evidence that our universe exhibits mathematical fine tuning, and argue that there is some preliminary evidence that the universe is anthropically fine-tuned. However, one of the points we hope to underscore is that making the case for anthropic fine tuning is often more difficult than both its supporters and detractors seem to believe.

\subsection{Evidence for Mathematical Fine Tuning}

One way to understand the lack of empirical data for the existence of mathematical fine tuning is by an appreciation of its historical development. As indicated above, early speculation of the idea of astrophysical fine tuning, from Pythagoras to Dirac (1938), focused on the existence of mathematical fine tuning. Modern speculation on fine tuning begins in the 1960s, when there was a transition to an interest in anthropic fine tuning with the writings of Carter, Carr and Rees, et al. Failure to appreciate the distinction between mathematical and anthropic fine tuning encourages the error of seeing the history of thinking about fine tuning as a seamless continuity. Once this distinction is appreciated, it is clear that thinking about fine tuning has two distinct phases. (Although as we have noted above, some of the modern authors run these two types of fine tuning together, so the two distinct phases here are not always easy to see.) Historically, the decisive point here is perhaps Dicke (1961) who demolished Dirac's largenumber hypothesis. Dirac's hypothesis was, in turn, based upon the numerological speculations originating with Weyl and, as noted, widely promoted by Eddington. Dicke's argument is pivotal here because he employed a version of anthropic fine tuning in the argument against Dirac's case for mathematical fine tuning. 
Dicke's great-and in our view still deeply underappreciated, at least in some philosophical circles - achievement was to effectively put a stop on the type of numerological speculation in the vein of Weyl, Eddington, and Dirac, by showing that the apparent numerical coincidences are effective consequences of the observation selection. While Dirac asked how improbable it is to have the Newtonian gravitational constant $G$ being in a particular numerical relationship with, say, the age of the universe $t$, and consequently (in)famously predicted that the strength of gravitation decreases with time ( $G$ $\propto t^{-1}$ ), Dicke simply noticed that other possible values of $G$ and $t$ are incompatible with our existence as intelligent observers. In other cosmological epochs, such an improbable relationship might not hold (being substituted with something 'more natural'), but nobody can ask the question, since those epochs are not conductive to the existence of life and observers. Thus, Dicke was able to cut short all talk about numerical 'conspiracies' and 'mysterious coincidences' by pointing to a piece of practical astronomer's and physicist's daily bread-and-butter work: selection effects. In a sense, Dicke's explanation of 'anthropic' coincidences is similar to Darwin's achievement of explaining the apparent teleology of nature by evolution through natural selection. Darwin pinpointed the ubiquitous mechanism for producing apparently finely crafted particular traits of living creatures, as noticed by products of the same mechanism, such as ourselves, thus providing a 'universal solvent' for getting rid of supernatural or overly complex 'skyhook' explanations (Dennett 1995). Dicke similarly perceived that the cosmological and stellar evolution themselves provide for generation of the noted relations without any additional skyhooks: large number coincidences are observed only because any conceivably different values to such dimensionless quantities would be incompatible with our existence as intelligent observers (and consequently the relationships are only approximate). It is especially important to understand that Dicke's approach has been corroborated by all subsequent research in astrophysics and cosmology, and in particular by the clear-cut falsification of predictions of Dirac's and similar theories (e.g. Hellings et al. 1983; Damour, Gibbons, and Taylor 1988).

It is worth noting that the conflation of these two types of fine tuning is not limited to merely the detractors of the idea of astrophysical fine tuning, nor is it correct to say that all discussion of mathematical fine tuning has disappeared in modern (that is, post 1960s) discussion of fine tuning. For instance, as mentioned briefly above, the monograph of Barrow and Tipler (1986)—what Klee aptly describes as the 'Bible' of anthropic reasoning - is not entirely innocent of this confusion. What this suggests is that all those involved in this debate should be on guard against conflating the two types of fine tuning.

This little history lesson goes some way to explaining why mathematical fine tuning has fallen out of favour. To this, we should add that contemporary physical evidence provides little support for their hypothesis: we now know that the numerical regularities in, for instance, the value of fine-structure constant are very approximate and cannot be traced to a solution of a simple quadratic equation, as Eddington hoped. The number of particles in the universe is determined by complicated interplay between cosmological parameters (density $\Omega$ and cosmological constant $\Lambda$ ) and Big Bang physics, which cannot be formulated in a simple arithmetical manner, etc. The project of 
unification of forces is, if anything, livelier than in Eddington's day, but in its modern clothes of, say, string theory, it is based upon arcane mathematical physics in which any traces of Eddington numerology have long vanished.

To say this is of course not to deny the possibility that string theory or some other new physical theory might not qualify as a case of mathematical fine tuning. Rather, it is to say that nothing on the horizon suggests that string theory or its competitors are mathematically fine-tuned. (We will discuss possible exceptions to this claim below.)

\subsection{Empirical Evidence for Anthropic Fine Tuning}

It is our contention that, unlike purported cases of mathematical fine tuning, the empirical case for anthropic fine tuning is still very much alive and well. As noted, however, often both supporters and detractors make grandiloquent statements of the type 'phenomenon $\mathrm{X}$ is/is not a manifest example of fine tuning', while the details of serious astrophysical observational and theoretical effort are completely lost. We hope to illustrate what is involved by considering two cases. (A more detailed consideration of all purported cases of anthropic fine tuning would probably require a book-length study.)

Our first case is the conjecture that galactic density is a case of anthropic fine tuning. It is clear that there is at least some relation between galactic volume and mass, and the possibility of the existence of sentient observers. When galactic density is too great, galaxies will tend to collapse into their gravitational centre; on the other hand, when the density is too low, stars may never form. Either case is obviously incompatible with the existence of observers. But how delicate is this balance?

In order to answer this, let us begin with a very general point: if one wishes to draw conclusions dealing with the history and philosophy of science, one would do very well to first ascertain the fine details in each particular discipline that rarely find their way into textbooks and other polished presentations, for here is where one will often find 'awful truths'. One of the 'awful truths' of astrophysical science is that our knowledge of the mass and size of galaxies is highly uncertain to an order of magnitude or greater. Even in our present state of ignorance, however, it is clear that most of the matter in any galaxy is located in the dark halo, and that everything visible — technically speaking, what is contained within the Holmberg radius of a galaxy, typically about $20 \mathrm{kpc}$ (kiloparsecs, $1 \mathrm{kpc}=3.09 \times 10^{19} \mathrm{~m}$ ) -is just the proverbial 'tip of the iceberg', never more than about $20 \%$ of the size and $10 \%$ of the mass of the whole (and often much less). It is a daunting observational task to determine these parameters exactly, and it is not disgraceful to admit that we still do not know the mass of even our own galaxy (the Milky Way)—not to mention other galaxies—with any great deal of precision.

Thus, even if there is a claimed anthropic 'prediction' of values of these quantities, it seems clear that we cannot at this stage justly assess the value of such a prediction. However, this has not stopped both proponents of anthropic fine tuning (Carr and Rees 1979) and opponents (Klee 2002) from speculating about this matter. Recently, Klee used the value of $10^{41} \mathrm{~kg}$ to represent the mass of the Milky Way, which is about $5 \times 10^{10} \mathrm{M}_{\odot}\left(\right.$ Solar masses, $1 \mathrm{M}_{\odot}=1.989 \times 10^{30} \mathrm{~kg}$ ), which is very far off the mark, 
being certainly too small. We shall quote only a handful out of a host of measurements performed in the last decade, in order to show the complexity of the problem. By considering the Milky Way's outer satellites, Kulessa and Lynden-Bell (1992) obtained a mass of around $1.3 \times 10^{12} \mathrm{M}_{\odot}$ within $230 \mathrm{kpc}$. The inner part of the Galaxy is accessible to measurements via the high-velocity stars; using this technique, García Cole et al. (1999) estimate the mass within $45 \mathrm{kpc}$ to be $5 \times 10^{11} \mathrm{M}_{\odot}$, still an order of magnitude larger than Klee's value. Kochanek (1996), using several methods concordantly, obtains similarly $(4.9 \pm 1.1) \times 10^{11} \mathrm{M}_{\odot}$ out to the galactocentric distance of $50 \mathrm{kpc}$. Finally, Wilkinson (2000) suggests $1.9 \times 10^{12} \mathrm{M}_{\odot}$ (with a lower error of 1.7 and an upper error of $3.6 \times 10^{12} \mathrm{M}_{\odot}$ ) with the scalelength of $170 \mathrm{kpc}$. Similar data are available for other giant spiral galaxies comparable to the Milky Way in luminosity and other properties. The best studies so far have been performed by Dennis Zaritsky and his coworkers, on the basis of observations of satellite galaxies. Zaritsky and White (1994) claim the average mass of a spiral galaxy to be $1.5-2.6 \times 10^{12} \mathrm{M}_{\odot}$ within $200 \mathrm{kpc}$. In continuation of that study, Zaritsky et al. (1997) confirm the average mass to lie in excess of $2 \times 10^{12} \mathrm{M}_{\odot}$ ' with strong dynamical indications that dark haloes extend to more than $400 \mathrm{kpc}$ ! In any case, Klee's fiducial radius of about $2 \mathrm{kpc}$ (about $6 \times 10^{19} \mathrm{~m}$ ) is astonishingly wrong (being, for instance, more than four times smaller than our own distance from the Galactic centre!). Thus, one of the largest inaccuracies of 'anthropic' predictions in Klee is simply a consequence of the two orders of magnitude error on his part. Carr and Rees can be forgiven an error like this, since most of this modern galactic astronomy was completely unknown in the late 1970s, but should not be overlooked by Klee, who writes in 2002, well after what, in effect, has been a revolution in our understanding of the nature of galaxies.

The same imprecision may be applied to quantities such as stellar mass and length, since (if we forsake vulgar heliocentrism!) these quantities are not unmistakably or fairly represented by their Solar values, which Klee uses to check the accuracies of the anthropic predictions. This is not to say that the values thus obtained would be very different from the value Klee uses (Solar values); and it is even probable that the discrepancy with the anthropic predictions would be even larger in these cases (since, for instance, the average stellar mass is obviously lower than $1 \mathrm{M}_{\odot}$, no matter which stellar mass function turns out to be the best). But these empirical findings of astrophysics must be taken into account if one purports to discuss astrophysical quantities seriously. Not to do so would usually bias us towards (and not against) our privileged position in the universe! The upshot of this seems to be that on the question of the anthropic fine tuning of galactic mass, the considered judgement here is that 'the jury is still out'. After all, a necessary condition for making the judgement that galactic mass is fine-tuned is that we know with some precision the actual mass of the galaxy!

Our second case for further scrutiny is the (in)famous story about fine tuning associated with carbon-12. While this presents us with a whole nest of interesting and controversial issues, we shall, for the sake of brevity, confine ourselves to a single point: the use of contemporary research into the anthropic significance of the nuclear resonance in light elements. Here again, Klee provides a useful negative example. ${ }^{5} \mathrm{He}$ concludes this section (6) of his paper with the following remark: 
The quantitative details are thorny and depend on the specific model of stellar structure used, but the width of the window of compatibility appears to be wide enough to undermine any claim that we have a case of fine tuning on our hands. (Klee 2002,347)

Klee attempts to support this contention by citing empirical research—but herein lies a serious problem; there is a sizeable subfield of anthropic research, and there have been several important references dealing with it (Livio et al. 1989; Pochet et al. 1991; Hogan 2000; Oberhummer, Csoto, and Schlattl 2000). Unfortunately, Klee cites just one of these modern studies, and the oldest one at that (Livio et al. 1989), the results of which (as Klee correctly sees) could be called critical toward the conventional anthropic view of these nuclear resonances. This selective approach creates the misleading impression that modern research has somehow disproved Hoyle, Barrow, and Tipler, and others who perceive something important in these data, and in need of an explanation. Other studies (and subsequent to Livio et al. 1989) report different results, and much more in the spirit of the idea that reality is anthropically fine-tuned. For example, Oberhummer, Csoto, and Schlattl emphasize that:

[A] change of more than $0.5 \%$ in the strength of the strong interaction or more than $4 \%$ change in the strength of the Coulomb force would destroy either nearly all C or all $\mathrm{O}$ in every star ... Therefore, for the above cases the creation of carbon-based life in our universe would be strongly disfavoured. The anthropically allowed strengths of the strong and electromagnetic forces also constrain the Higgs vacuum expectation value ... and yield tighter constrains on the quark masses than do the constrains from light nuclei ... Therefore, the results of this work are relevant not only for the anthropic cosmological principle ..., but also for the mathematical design of fundamental elementary particle theories. (Oberhummer, Csoto, and Schlattl 2000, 90)

What should we make of this fact that a change of ' $0.5 \%$ in the strength of the strong interaction or more than $4 \%$ change in the strength of the Coulomb force would destroy either nearly all $\mathrm{C}$ or all $\mathrm{O}$ in every star'? This is perhaps not 'fantastical precision', as Klee claims, but 'fantastic precision' is anyway a straw person. If the intent of the critics was to show that the universe was mathematically fine-tuned perhaps (let us grant for the sake of the argument) then one might hope that the numbers would yield a more numerologically appealing result than a difference of $0.5 \%$ and $4 \%$, but this is not what Oberhummer, Csoto, and Schlattl are attempting to draw our attention to. It is the fact that the universe appears to be designed for human life, since even fairly small changes in its fundamental properties (compared with prima facie infinite possible interval of these real numbers) would mean that human life would not be possible.

Are there sufficient data to prove the existence of any significant anthropic fine tuning? As we argued above, it is not clear that the judgement that galactic mass is finetuned can be supported at this stage. On the other hand, the other example we considered does lend some weight to the idea that our universe is fine-tuned, for the narrow range of values in which nuclear synthesis might fall in itself seems sufficient to warrant a call for explanation. It may be objected that this in itself may seem insufficient for supporting a judgement that our universe is anthropically fine-tuned, but there are other instances of apparent fine tuning to our universe that themselves seem to call for an explanation: for example, the fact that human life is unimaginable if the universe 
had contained only a highly diluted hydrogen gas, or if it had recollapsed before the temperature anywhere had dropped below $10000 \mathrm{~K}$, which corresponds to early expansion speeds different from the actual for only (plus or minus) one part in $10^{10}$. Likewise, the universe containing only black holes (and thus extremely inhospitable for anything remotely similar to human life) is statistically more probable from the observed configuration of matter for a factor containing a stupendous double exponential (e.g. Penrose 1989; Ćirković 2003). This is not the place to review the empirical details, however, we believe that at this stage, the empirical evidence incontrovertibly supports the following:

The Anthropic Fine Tuning Conditional: It appears that if the values of the fundamental constants of the universe are assigned randomly, then the chances of human life developing in this universe are astronomically small.

We should remind ourselves that this is understood as an observational datum, which is distinct from the explanatory task of accounting for the anthropic fine tuning conditional. To this, we now turn.

\section{Explaining Astrophysical Fine Tuning}

In this section, we consider the theoretical or explanatory component of fine tuning: How should we explain the existence of anthropic fine tuning? We will consider six explanatory possibilities: mere coincidence explanation, the Design explanation, the lesser designer explanation, the multiverse explanation, the 'mere appearance explanation', and the no-explanation response.

\subsection{Coincidence Explanation}

Let us think first of the idea that anthropic fine tuning can be explained naturalistically as 'mere coincidence' or 'mere luck'. This explanation accepts the observation that it is highly unlikely that the universe is hospitable for human life and adds that what this shows is something that we already know: small probability events sometimes occur. Imagine the chances are 50/50 that our universe is hospitable for human life. It would hardly seem inappropriate (other things being equal) in this case to appeal to anything deeper than 'the luck of the draw' to explain why it is that our universe is hospitable for life. Similarly, the coincidence explanation says that if the chances are much less likely than 50/50, this does not change the logic of the explanation.

The difficulty in accepting this sort of explanation for our universe is that the probability of it being hospitable for human life is so much lower that there seems to be something deeply unsatisfactory about this sort of explanation. Writing in the authoritative Annual Reviews of Astronomy and Astrophysics, Sir Fred Hoyle succinctly summarized the line of argument against the suggestion that 'mere luck' offers much of an explanation here:

Suppose you were a superintellect working through possibilities in polymer chemistry. Would you not be astonished that polymers based on the carbon atom turned out 
in your calculations to have the remarkable properties of the enzymes and other biomolecules? Would you not be bowled over in surprise to find that a living cell was a feasible construct? Would you not say to yourself, in whatever language supercalculating intellects use, 'Some supercalculating intellect must have designed the properties of the carbon atom, otherwise the chance of my finding such an atom through the blind forces of nature would be less than 1 part in $10^{40000}$. Of course you would, and if you were a sensible superintellect you would conclude that the carbon atom is a fix. (Hoyle 1982)

According to Hoyle, then, the point is that this 'fix' indicates a grand architect of at least the fundamental constants and values of our universe. It is worth noticing that the claim here is that the universe has been designed to allow life rather than being designed to exhibit mathematical orderliness. Hoyle's argument has a negative and positive aspect: the former is the rejection of the idea that mere coincidence or luck is sufficient to explain the properties of the carbon atom; the positive aspect is the idea that properties of the atom are the results of an intentional process. Let us concentrate first on the negative aspect of Hoyle's argument.

Consider the following response to Hoyle's line of thought:

How does the low probability affect the argument? Why not simply chalk up the properties of the atom and thus our existence to fortuitous coincidence? Granted that if the properties of the carbon atom were significantly different life would not have developed. But so what? Our universe could simply be a series of fortuitous coincidences.

It is clear that this response points to a logical possibility, but we should be clear that this is an explanation about how we should understand anthropic fine tuning. As such, it should be subject to the usual canons of acceptable scientific explanations. Consider that if we flip a coin and it comes up 'heads', we might make a conjecture that this is merely a random event-it could just as easily have come up tails. The same could be said if we flip the coin another million times, and it comes up heads each time-we might conjecture that the appropriate theory to explain this is a mere coincidence: after all, small probability events do occur. On the other hand, it would seem a colossal failure of scientific imagination not to at least consider other possible explanations here, e.g. that what appears to be a massive coincidence is actually the result of some underlying causal process (e.g. a magnetized coin landing on a magnetic table). The same point applies with respect to anthropic fine tuning: an appeal to coincidence is one possible explanation, but the probability of an event such as our universe seems so unlikely that it would be a colossal failure of scientific imagination not to look for other possible explanations.

Indeed, we can make a stronger point here. Imagine you observe the coin landing heads a million times, and you seek to explain this. You rule out every causal explanation for this event you can think of: you check to see if the coin is magnetized, a trick coin where both sides are heads, and so on. Your best efforts fail to discover any causal explanation for why the coin has landed heads a million times. Now, you are forced to bet on something you value highly (e.g. your life) on one of two hypotheses: that the coin will land heads the next 10 tosses in a row or that tails will appear at least once. If you think the coin will land randomly, then you should bet on the second hypothesis: 
that it will land tails at least once. After all, the odds of being wrong are 1/1024, on the assumption that the coin lands randomly. Which hypothesis would you bet on, given that the coin has previously landed heads a million times? Presumably, most of us, because of epistemic humility, would bet on the first hypothesis and not the second, for it would seem more likely that we have not been able to figure out the reason for the coin's regular behaviour rather than thinking it was merely a matter of coincidence that it landed heads a million times in a row. If we accept the analogy, then it seems that we ought to reject the mere coincidence hypothesis, even if we cannot discover the explanation for the anthropic fine tuning. We cannot assume a priori that the universe will in fact prove comprehensible to us (Walker 2004).

Sometimes, the objection is thought to be strengthened by a seemingly plausible line of anthropic reasoning. Thus we might imagine someone arguing as follows:

All that the fact that the universe is exceedingly unlikely to be hospitable for human life shows is that it is unlikely we would be around to ask questions about the nature of our universe. In other words, what this tells us is that any universe where observers exist is one that will look 'miraculous', but it need not be literally miraculous. Imagine a coin that connected to a doomsday device. Every time it lands heads nothing happens, if it lands tails the doomsday device kills all sentient life. We should not be surprised to find that it lands a million times heads, since any other outcome would mean that we are not around to observe the outcome.

Put in these terms, the elementary fallacy is perhaps obvious. Clearly, it is not surprising that we are around when we ask the question of why the coin landed heads a million times. But landing a million heads in a row is surprising, and seemingly in just as much need of explanation, as when there is no doomsday connected to the coin. In either case, the probability of the coin landing heads a million times seems astronomically small, and so seems to call for some explanation. In other words, the two cases are identical in that they beg for some explanation of the coin landing heads a million times. The fact that there is a doomsday device connected to the coin's outcome in the second case should not affect the perceived need to explain this.

\subsection{Designer Explanation}

Given that our universe being hospitable for human life is far less likely than having a fair coin land heads a million times in a row, we may not find the mere coincidence hypothesis particularly satisfying. At a very minimum, we should consider the alternatives before settling on it, and so let us turn to Hoyle's positive suggestion: that our universe should be seen as a 'fix', that the intentions of a Designer explain our universe. One obvious candidate for Designer status is the God of traditional theism: an omnipotent, omniscient, eternal, and morally perfect being. Putting aside for the moment the scientific credentials of such a hypothesis, one might wonder whether it holds up under theological scrutiny. After all, if God is a perfect being, then surely he could have done a much better job of designing this universe. For example, should we expect that the universe might exhibit mathematical and perhaps moral fine tuning (e.g. that this is the best possible world), if God is the Designer of the universe? 
The following considerations show that this objection could not be decisive. It seems obvious God could have made the universe such that it is overwhelmingly obvious that there was a designer at work. For instance, God could have engraved or marked every stone, every animal, every plant, etc., with the word ' $\mathrm{God}^{\mathrm{TM}}$ '. We can easily imagine scientific investigation eliminating any possibility of hoax, e.g. if radioactive dating revealed that the engraving on many rocks predates the evolution of life on this planet, then we would have pretty solid evidence of a designer at work in our universe. Or God could have made our universe a PNU. So, clearly then God has in his powers the ability to flaunt the fact that He designed the universe. But while this sort of obviously designed universe is within God's ability to make, God has moral reasons for not making the universe in this way. Specifically, God did not design the universe this way because He wanted to make the universe, where possible, ambiguous between a secular and theistic interpretation; so that we could freely choose to embrace Him or not, and that we may freely will to perform good or bad acts. As Murray argues:

... it seems clear that fully robust and morally significant free will cannot be exercised by someone who is compelled by another in the context of a threat... . [I]f God does not remain 'hidden' to a certain extent, at least some of the free creatures He creates would be in the condition of being compelled in the context of a threat and, as a result, such creatures could not exercise their freedom in this robust, morally significant manner. (Murray 1999, 243)

A universe that exhibited overwhelming design qualities would not allow any free choice in the matter of whether to believe in God, or whether to perform good or bad acts in the absence of a threat. So, it would have been improper for God to make the universe in a way that exhibits too much mathematical fine tuning, since He would not have remained sufficiently 'hidden'. The fact that there is evidence of anthropic fine tuning is explained by the fact that God wants to lay clues to His existence, but He does not want to make the evidence so overwhelming that it forces everyone to believe in Him. (On the other hand, He did not want to make the evidence against His existence overwhelming either.)

So, if this reply is successful, there is no reason to suppose that God must have created the universe in a way that exhibits more design than we in fact observe. In other words, the moral here is that an appeal to the Design Hypothesis does not logically commit one to the idea of mathematical fine tuning of the universe; the appeal to anthropic fine tuning alone may be sufficient to motivate the Design Hypothesis. On the other hand, if the universe does exhibit mathematical fine tuning, then this may lend some support to the Design explanation (see below). And clearly, Hoyle's idea that we should exhibit the anthropic fine tuning to the work of some agency leaves considerable latitude as to the exact nature of this agent. In addition to the traditional JudeoChristian God, several other possibilities suggest themselves, including Leslie's divinity who takes a more 'hands-off' management style with the universe (Leslie 1989), much like the Demiurge of Plato and Aristotle.

The advantageous and disadvantageous of the Designer hypothesis are fairly obvious. On the one hand, it would allow us to forgo having to cite infinitesimally small probabilities to explain our existence. On the other hand, considerations like Occam's 
razor might argue that we ought to reject such appeals. It is interesting to speculate whether one should, if only given the two options we have considered thus far, opt for the mere coincidence or the Designer hypothesis. It is easy to say that we ought to prefer the coincidence hypothesis, since we find the thought of some Designer of our universe very unlikely, and that we know that small probability events do occur. However, one may wonder whether there may be some failure of imagination here: imagine watching the doomsday coin being flipped and being offered only one of two choices to bet on after each coin toss: either the coin will land randomly or there is an agent at work controlling how it lands. Initially, we might think that the event is random and that there is no need to appeal to the work of a Designer. After it lands heads a million times, one may well wonder whether the Designer hypothesis is correct.

\subsection{Lesser Designer Explanation}

A variant on the previous line of argument is Linde's suggestion that our universe is a 'basement' universe created in another universe. The argument is similar in that it appeals to the idea of an intentional designer of our universe; the main difference is that the designer is not one of traditional theism, but merely the workings of an advance scientist (a 'lesser' designer). The physics of creating basement universes is of course controversial, but the point here is simply that given the possibility of basement universes, we do not necessarily need to think of the Designer in a traditional theistic manner. Rather, it seems we could think simply of very proficient scientists-scientists who are capable of making basement universes in their lab. Interestingly, Linde has recently speculated that if our universe is a basement universe, then we might find a message from the creators of this universe somehow inscribed into the fundamental physics of our universe (Holt 2004). Linde does not say in detail what we should be looking for, but the basic idea seems to be that the superscientists would create universes which are both hospitable for life and somehow inscribed a message in the fabric of the created universe- this being the only way that the creators could communicate with the denizens of the basement universe. In the terms developed here, the idea seems to be that the basement universe might be anthropically fine-tuned as well as mathematically fine-tuned. Given these assumptions, Linde's reasoning on this point seems sound: if there are superscientists creating basement universes who hope to provide evidence of their own existence, then anthropically and mathematically fine tuning the universe might be a plausible strategy to pursue.

As we noted above, presently there is not much that might be considered empirical support for the idea that the universe is mathematically fine-tuned. However, it is worth considering what might follow if future developments in physics lend support to the idea that the universe is mathematically fine-tuned. Conceivably, such developments might lend some support to the Designer or the lesser designer hypotheses. It might be argued, for instance, that a Demiurge or a scientist creating basement universes might hope to mould the universe with such an aesthetic in mind, perhaps, as Linde speculates, to communicate something to us. But would mathematical fine tuning necessitate either of the design hypotheses? The short answer is no because it 
would depend in part on our estimation of other possible explanations including naturalistic explanations for mathematical fine tuning. There are parallels in the biological world where one might think that mathematical fine tuning is at work, e.g. the Fibonacci number series characterizes a number of biological phenomena (Ball 2001). Biologists typically look to naturalistic explanations for this observation in terms of developmental and resource efficiency (Ball 2001). Scientists have also investigated three species of North American Cicada that spend almost their entire lives underground in a nymphal stage emerging every 13 or 17 years (Lloyd and Dybas 1966a). The fact that the emergence period converges on prime numbers has not gone unnoticed, and various naturalistic explanations have been offered in terms of thwarting parasites, predators, or competitors (Lloyd and Dybas 1966b; Gould 1977; Cox and Carlton 1998). Some theorists have rejected such explanations, attributing the prime number periodicity to mere coincidence, that is, that it is merely a coincidence that the period of the life cycles of these three species should converge on two prime numbers (Hoppensteadt and Keller 1976). The point then is that when investigating the apparent mathematical fine tuning of biological phenomena, biologists tend to look for naturalistic explanations rather than appealing to the intention of a Designer (or designer) concerned to make biological phenomena conform to some mathematical design. Similarly, if the universe turns out to be mathematically fine-tuned, it would not be a foregone conclusion that we must appeal to the intentions of a Designer (or designer); rather, at a minimum, we would need to consider whether some naturalistic explanation could be found. In any event, the point is really mute, since there is no evidence (at present) that the universe is mathematically fine-tuned.

\subsection{Multiverse Explanation}

There are a significant number of scientists (and philosophers) who recognize the validity and significance of anthropic fine tuning, but they do not see anything even remotely similar to the Design argument in it. Indeed, it is arguable that the core of the anthropic reasoning, as noticed by many physicists (like J. Richard Gott, Fred C. Adams, etc.,), is in fact naturalistic and anti-teleological: it accepts the anthropic fine tuning of our cosmological domain as a puzzling empirical fact in need of an explanation that does not appeal to the intentions of a Designer (or lesser designer). One such explanation is suggested by advances in physics: if there is an ensemble of universesalso called the multiverse-with a suitably varying range of properties (and current quantum cosmological models, like Linde's chaotic inflation (e.g. Linde 1986, 1990), do suggest that that is the case), then one would naturally expect some of these universes to be just right for life. Even if such universes are in a small minority, because of an 'observation selection effect'-the lifeless universes, where the constants are 'wrong' contain no observers, and cannot be observed-such a theory can predict that we should observe precisely what we do in fact observe: a universe that appears to be reasonably anthropically fine-tuned. This does not happen because the underlying theory is fine-tuned, or incorporates anything a priori improbable, but because an observation selection effect guarantees that we observe a very atypical part of the whole 
of physical existence. In other words, the embedding of an atypical (or fine-tuned) region of the entire reality into the larger whole provides a perfect anti-teleological and naturalistic explanation of our (a priori improbable) observations (this was emphasized by the late Robert Nozick as his 'principle of fecundity'; Nozick 1981). ${ }^{6}$ Physicists and astronomers have been familiar with the observational selection effect for a long time, some aspects of it (e.g. Malmquist bias in astronomy ${ }^{7}$ ) being the subject of studious and detailed mathematical modelling. In terms of our coin-tossing analogy, if trillions of coins are being tossed simultaneously we should predict that sooner or later, some would have a series of one million heads in a row.

The question arises, if scientists have been aware of observational selection effects for so long, why has it suffered relative neglect in the philosophy of science? While not directly addressing this issue, Klee suggests a possible reason when he writes:

I believe it is a mistake to see any virtue in trying to construct formal Bayesian probability arguments in the present context. To his credit, Leslie does not do so. He formulates his argument as an abductive one in which the mathematical data on the allegedly fantastically narrow intervals of variance are not used in a technically formal way. (Klee 2002, 352)

Klee here is referring to Leslie's theistic argument in Universes (Leslie 1989). According to Klee, the problem with trying to formulate something stronger than Leslie's inference to the best explanation argument is that there is an insurmountable problem arising from issues involving measurement scales. While we cannot explore the issue in detail here (and Klee does not pursue it either), the basic complaint is that the interval of variance compatible with hydrocarbon life can be made to seem larger or smaller depending on one's measurement scale. So, according to Klee, because of this, there is no hope of quantifying the argument with greater precision, and thus, nothing stronger than an argument to the best explanation can be made. This in turn suggests that there is a limited amount that philosophers and scientists can do to clarify and quantify the argument. Whether this is ultimately so remains to be seen. An antidote for such pessimism can be found in Bostrom (2002) (which is not discussed by Klee). It points out immediately that cosmology is fundamentally incomplete without taking into account the necessary 'anthropic bias': conditions we observe in fundamental physics (as well as in the universe at large) that are atypical and require an explanation. Furthermore, Bostrom has constructed a Bayesian argument precisely of the sort Klee says there is no virtue in formulating. If this line of argument can be maintained, then not only is Bostrom's approach to anthropic coincidences superior to Leslie's, but it offers a chance of obtaining solutions to many serious problems in the philosophy of cosmology and physics. The central piece of this approach is the Observation Equation (Bostrom 2002, 173), which subsumes seemingly vague assumptions and observational selection criteria in full mathematical rigour. Moreover, the last chapter of Bostrom's monograph gives an example how the very same Bayesian apparatus can be applied to both astrophysical fine tuning and to such issues as traffic planning and the origin of the thermodynamical arrow of time. In any event, if something along the lines sketched by Bostrom is correct, then it points the way philosophers of science might pursue the question of anthropic coincidences in a naturalistic and rigorous manner. 
One of the obvious advantages of the multiverse explanation is that it provides a naturalistic explanation that does not invoke the intentions of a Designer (or lesser designer); nor does it appeal to mere good fortune. The obvious disadvantage of the multiverse explanation is that it must invoke an ontology which, as the old joke goes, only an American could afford. Again, the issue of Occam's razor would have to be factored in. However, it is worth noting that there are independent reasons for thinking that the multiverse hypothesis is viable, independent, that is, of its explanatory role in explaining anthropic fine tuning, e.g. the multiverse plays a significant and important role in much-investigated physical ideas (namely those of inflation and quantum cosmology). For instance, the much talked-about 'anthropic landscape' of the most investigated M-theory presents not an optional, but a very vital ingredient necessary to explain the ways of compactification of additional dimensions, as well as the observed value of vacuum energy (e.g. Banks, Dine, and Motl 2001; Freivogel and Susskind 2004; Aguirre and Tegmark 2005). So, one reason to favour the multiverse explanation of anthropic fine tuning is that it fruitfully accommodates both quantum cosmology and fundamental physics (embodied in M-theory, for instance), and even thermodynamics and topological quantum field theory.

\section{5. 'Anthropic Fine Tuning Is Apparent, not Real' Explanation}

The final two explanatory stances we shall consider reject different parts of anthropic fine-tuning reasoning. The first rejects observational claims to the effect that our universe is fine-tuned, and the second rejects the call to explain this observation. Let us take these in turn. Some theorists, for example, maintain that once we have an adequate physical theory, we will see that our universe could not have been other than it is. Once the default view for physicists of the naturalistic bent, it has gradually lost most of the adherents in face of the overwhelming empirical evidence for anthropic fine tuning, combined with the fiasco of all simple schemes of physical unification (e.g. Einstein's and Schrödinger's theories of 'unification' of gravitation with electromagnetism) which were at least partially motivated by such considerations; witness the famous Einstein quote: 'What really interests me is whether God had any choice in the creation of the world.' In fact, the advancement of science actually increases theoreticians' latitude, contrary to what this view entails (less and less freedom in our choice of underlying parameters consistent with the empirical world). However, this view should still be taken seriously; it has been recently invoked, for instance, by Smolin (2004). If this is the case, then we would have to reject some of the claims made above, for example, that the properties of the carbon atom are improbable, and the claim that it is much more probable that our universe might have ended up as a lifeless collection of black holes. In terms of our coin analogy, imagine we did not know about magnetism and we watch with amazement as a coin land heads a million times in a row. Once we discover the magnetic properties of the coin and the table, what seemed like a huge improbability turns out to be a virtual certainty: the coin could not land tails because of magnetic polarity. If we accept the analogy then the underlying physical theory will explain why the universe must be hospitable for life. In fact, this sort of development is a reasonably 
common occurrence in science, e.g. Copernicus, Kepler, and Newton combined to explain how the seemingly tortured course of the planets through a series of epicycles had much more unity than was apparent to early theorists. That said, there is nothing on the horizon that would suggest string theory will develop in such a way as to show that our universe could not have developed otherwise (it is perhaps not completely fortuitous that Smolin is the main proponent of the minority alternative to the string theory, namely quantum loop gravity, which does not require the multiverse in the sense that string/M-theory does). On the contrary, it needs to be re-emphasized that the freedom in 'universe construction' has generally increased in recent decades, a trend antithetical to this view. Still, it is worth keeping an open mind about different possible developments.

\section{6. 'No Explanation' Response}

A final explanatory stance is to reject the project of attempting to provide a scientific explanation for anthropic fine tuning. Here, one may accept that the universe appears to be fine-tuned but reject the idea of attempting to provide an explanation for this. For example, it might be thought that the various explanations offered belong to the domain of metaphysics rather than science, or that the cosmological parameters should be accepted as 'brute facts'. This sort of response is likely to be driven by particular conceptions drawn from the philosophy of science, for example, one reason to endorse this view might be based on the idea that empirical verification or falsification is the hallmark of science, and these explanations are not open to infirmation or confirmation; hence, they are not scientific explanations. Certainly this is not the place to decide on this philosophy of science issue; rather, we mention it to point out simply that acknowledgement of astrophysical fine tunings does not necessitate looking for a scientific explanation-this would require further argument. On the other hand, it seems likely that scientists will not be able to resist theorizing about this matter, given the profundity of the question. Forbidding the discussion by fiat has never-as history of thought shows us-ever solved a problem, nor what is considerably more important here, has it ever prevented people from inquiring. After all, providing an explanation for anthropic fine tuning may go a long way to answering the question of why we are here.

\section{Conclusion}

We wish to conclude by emphasizing three points. First, anthropic fine tuning is analytically completely divorced from Eddingtonian numerology and other claims that the universe is mathematically fine-tuned. Our best current understanding of the universe suggests that there is no relationship between the two, except the historical one. Any numerology is a counsel of despair, particularly today, when we may be on the verge of the completely unified theory of the dynamics of matter in nature. Eddington was a great intellect, but great people tend to make great mistakes; testify Newton's alchemy, Lord Kelvin's spiritism, or Schwinger's cold fusion. To criticize astrophysical 
fine tuning on the basis of weaknesses of naive numerological musings of the 1920s is about as appropriate as criticizing the concepts of modern chemistry on the basis of the flaws of the Ancient theory of matter consisting of earth, air, fire, and water. The anthropic principle has been perhaps overly criticized in recent decades, and often on flimsy enough grounds. It is, probably, a high time and good opportunity (offered, among other things, by some of the recent physical and cosmological studies quoted above) to pause, rethink, and try to reach a new and deeper level of discussion.

Relatedly, further work on the anthropic fine tuning is necessary in terms of both observation and theory. As we noted in Section 3, the observational evidence for some claims of anthropic fine tuning, such as galactic mass, are radically incomplete at this point, so additional work needs to be done in this area. On the theoretical side, we mentioned the beginnings of a naturalistic explanation in terms of a multiverse. This in itself shows the false dilemma in some versions of the Design argument. For instance, Hoyle, in the passage quoted above, assumes either that we reject the claim that the properties of the carbon atom are improbable, or the universe has a Designer (Hoyle's 'supercalculating intellect'). A naturalistic explanation like the multiverse allows us to reject Hoyle's dilemma, for then it is possible to accept the improbable nature of the properties of the carbon atom (anthropic fine tuning) while rejecting the idea of a Designer.

Finally, empirical evidence for the existence of anthropic fine tuning raises in a very real way the question for philosophers of science (and others) of when it is appropriate to explain some phenomena in terms of mere 'accident' or 'coincidence' and when the refusal to look for some deeper explanation is a sign of unscientific dogmatism. We have suggested that there is mounting empirical evidence that it is a priori extremely improbable that our universe should be hospitable for life. It may be true that most scientists do not find particularly appealing any of the alternate explanations for this, namely, the multiverse, Designer or lesser designer explanations. Still, it must be asked, is it not dogmatic and not in keeping with the spirit of science to attribute this merely to 'luck' without a serious exploration of the alternatives?

\section{Acknowledgements}

Many thanks to Ric Arthur, James W. McAllister, and two anonymous referees for a number of very useful criticisms and suggestions.

\section{Notes}

[1] Terminological points: we refer to the 'Design argument' with a capital to underscore the fact it invokes the intentions of a deity to distinguish it from possible confusion with the sorts of design that might be the product of some non-intentional process such as Darwinian natural selection. Furthermore, in this paper, we use 'naturalism' in the ontological sense that our universe has no deistic designer; we do not use it in its methodological sense in which 'naturalism' might mean the adoption of the scientific method.

[2] Of course, we need also to accept the fundamental importance of primes in the number theory which by definition holds in both our actual universe and the PNU. 
[3] On occasion, we will use the singular 'anthropic principle', although we acknowledge that this glosses over the fact that there are a number of different anthropic principles. See Bostrom (2002), chapter 3, for some discussion.

[4] It may be possible for other forms of intelligent life to develop under radically different conditions, e.g. science fiction is replete with intelligent beings of 'pure energy', there is a famous idea of Robert Forward about beings based on the nuclear force instead of electromagnetism, Fred Hoyle's 'black clouds', etc. Anthropic thinking is concerned with the conditions necessary for life as we empirically know it to develop.

[5] It is perhaps worth mentioning here that in our estimation, Klee is one of the best recent critics of astrophysical fine tuning. Although we are critical of some of Klee's results, we are in agreement with the general position of his article that serious work on astrophysical fine tuning requires studious attention to the empirical details. Klee has shown that some claims made by early researchers on behalf of 'mathematical fine tuning' are definitely not supported by contemporary empirical research. On the other hand, since Klee enjoins us to raise the bar on this research (not to engage in what he terms 'mathematical sharp practice'), it does not seem inappropriate to apply this same standard to his results.

[6] It is not beyond reason that such an understanding is present, for instance, in the passage quoted above from the summary of Oberhummer, Csoto, and Schlattl (2000); note the locution 'in our universe'.

[7] The difference between the average absolute magnitudes of stars (or galaxies or any other similar sources) in magnitude-limited and distance-limited samples, discovered in 1920 by K. G. Malmquist.

\section{References}

Aguirre, A., and M. Tegmark. 2005. Multiple universes, cosmic coincidences, and other dark matters. Journal of Cosmology and Astroparticle Physics 1: 1-3.

Ball, P. 2001. The self-made tapestry: Pattern formation in Nature. Oxford: Oxford University Press.

Banks, T., M. Dine, and L. Motl. 2001. On anthropic solutions of the cosmological constant problem. Journal of High Energy Physics 1: 031.

Barrow, J. D., and F. J. Tipler. 1986. The anthropic cosmological principle. New York: Oxford University Press.

Bostrom, N. 2002. Anthropic bias: Observation selection effects in science and philosophy. New York: Routledge.

Carr, B. J., and M. J. Rees Martin. 1979. The anthropic principle and the structure of the physical world. Nature 278: 605-12.

Carter, B. 1974. Large number coincidences and the anthropic principle in cosmology. In Confrontation of cosmological theories with observational data, edited by M. S. Logair. Dordrecht: Reidel.

Ćirković, M. M. 2003. The thermodynamical arrow of time: Reinterpreting the Boltzmann-Schuetz argument. Foundations of Physics 33: 467-90.

Cox, R. T., and C. E. Carlton. 1998. A commentary on prime numbers and life cycles of periodical cicadas. American Naturalist 152: 162-64.

Damour, T., G. W. Gibbons, and J. H. Taylor. 1988. Limits on the variability of G using binarypulsar data. Physical Review Letters 61: 1151.

Dennett, D. 1995. Darwin's dangerous idea. New York: Penguin.

Dicke, R. H. 1961. Dirac's cosmology and Mach's principle. Nature 192: 440-441.

Dirac, P. A. M. 1938. 'A new basis for cosmology. Proceedings of the Royal Society A 165: 199.

Eddington, A. S. 1923. The mathematical theory of relativity. London: Cambridge University Press.

Freivogel, B., and L. Susskind. 2004. A framework for the landscape. Physical Review D70: 126007.

García Cole, A., W. J. Schuster, L. Parrao, and E. Moreno. 1999. The mass of the Milky Way from a uvby- $\beta$ survey of high-velocity stars. Revista Mexicana de Astronomía y Astrofísica 35: 111-22. 
Gould, S. J. 1977. Ever since Darwin: Reflections in natural history. New York: Norton.

Hellings, R. W., P. J. Adams, J. D. Anderson, M. S. Keesy, E. L. Lau, E. M. Standish, V. M. Canuto, and I. Goldman. 1983. Experimental test of the variability of G using Viking Lander ranging data. Physical Review Letters 51 (18): 1609-12.

Hogan, C. J. 2000. Why the universe is just so. Review of Modern Physics 72: 1149-1161.

Holt, J. 2004. The big lab experiment: Was our universe created by design? Slate. Available from http://slate.msn.com/id/2100715; INTERNET.

Hoppensteadt, F. C., and J. B. Keller. 1976. Synchronization of periodical cicada emergences. Science 194: 335-37.

Hoyle, F., and N. Wickramasinghe. 1999. The universe and life: Deductions from the weak anthropic principle. Astrophysics and Space Science 268: 89-102.

Klee, R. 2002. The revenge of Pythagoras: How a mathematical sharp practice undermines the contemporary design argument in astrophysical cosmology. British Journal for the Philosophy of Science 53: 331-54.

Kochanek, C. S. 1996. The mass of the Milky Way. Astrophysical Journal 457: 228-43.

Kulessa, A. S., and D. Lynden-Bell. 1992. The mass of the Milky Way galaxy. Monthly Notices of the Royal Astronomical Society 255: 105-18.

Leslie, J. 1989. Universes. London: Routledge.

Linde, A. D. 1986. Eternally existing self-reproducing chaotic inflationary universe. Physics Letters B 175: 395-400.

Linde, A. D. 1990. Inflation and quantum cosmology. San Diego, CA: Academic Press.

Livio, M., D. Hollowell, A. Weiss, and J. Truran. 1989. The anthropic significance of an excited state of 12C. Nature 340: 281-84.

Lloyd, M., and H. S. Dybas. 1966a. The periodical cicada problem. I. Population ecology. Evolution 20: $133-49$.

Lloyd, M., and H. S. Dybas. 1966b. The periodical cicada problem. II. Evolution. Evolution 20: 466-505.

Manson, N. 2000. There is no adequate definition of 'fine-tuned for life'. Inquiry 43: 341-51.

Maynard-Smith, J., and Szathmáry, E. 1996. On the likelihood of habitable worlds. Nature 384: 107.

Mosterín, J. 2000. The anthropic principle in cosmology: A critical review. Acta Institutionis Philosophiae et Aestheticae (Tokyo, Japan) 18: 111-39.

Mosterín, J. 2005. Anthropic explanations in cosmology. In Logic, methodology and philosophy of science: Proceedings of the Twelfth International Congress, edited by P. Hajek, L. ValdésVillanueva \& D. Westerståhl. London: King’s College Publications.

Murray, J. M. 1999. Coercion and the hiddenness of God. In Philosophy of religion: The big questions, edited by E. Stump and M. J. Murray. Oxford: Blackwell.

Nozick, R. 1981. Philosophical explanations. Cambridge, MA: Harvard University Press.

Oberhummer, H., A. Csoto, and H. Schlattl. 2000. Stellar production rates of carbon and its abundance in the universe. Science 289: 88-90.

Penrose, R. 1989. The emperor's new mind: Concerning computers, minds, and the laws of physics. Oxford: Oxford University Press.

Penrose, R. 1991. The Emperor's new mind. New York: Penguin Books.

Pochet, T., J. M. Pearson, G. Beaudet, and H. Reeves. 1991. The binding of light nuclei, and the anthropic principle. Astronomy \& Astrophysics 243: 1-4.

Smolin, L. 2004. Scientific alternatives to the anthropic principle. In Universe or multiverse, edited by B. Carr. Cambridge: Cambridge University Press.

Walker, M. 2004. Skepticism and naturalism: Can philosophical skepticism be scientifically tested? Theoria 70: 62-97.

Weyl, H. 1919. Eine neve erweiterung der relativitätstheorie. Annalen der Physik 59: 101-33.

Wilkinson, M. 2000. The dark matter halos of galaxies: Masses and lensing properties. Observatory 120: 349 . 
Zaritsky, D., R. Smith, C. Frenk, and S. D. M. White. 1997. More satellites of spiral galaxies. Astrophysical Journal 478: 39-48.

Zaritsky, D., and S. D. M. White. 1994. The massive halos of spiral galaxies. Astrophysical Journal 435: 599-610. 